\title{
Analysis of Typical "Assembly-Components" Relationship Models in the Automobile Industry Chain
}

\author{
Zhu Minghao ${ }^{1}$, Zhang Zhibo ${ }^{1, *}$, Wu Xueqiao ${ }^{1}$ \\ ${ }^{1}$ School of Economics and Management, Beijing Jiaotong University, Beijing
}

\begin{abstract}
The research object of this paper is the model of correlation between automobile manufacturers and auto parts manufacturers in major automobile manufacturing countries of the world. After first analyzing the characteristics, advantages and disadvantages of typical "assembly-components" relationship models in US (Ford model), Germany (Volkswagen model), Japan (Toyota model) and China (Geely model), this paper explores the degree of correlation between companies involved in these typical models, and in the end draws conclusions and points out the deficiencies of the research.
\end{abstract}

\section{Research Background}

The development and manufacturing of automobiles is a complex systematic engineering process and automobile manufacturers are mainly focused on vehicle design and assembly, resource integration and supply chain management, while relying on independent external parts manufacturers to provide them with various other auto parts; and the main responsibilities of the parts manufacturers include detailed product design, manufacturing, inspection, quality assurance, timely delivery and after-sales service, and even the management and coordination of lower-level suppliers. Therefore, the core of the whole automobile industry chain is the upstream parts suppliers and downstream automobile manufacturers, and they jointly affect the development direction of the entire industrial chain.

Based on the above, it can be seen that in terms of helping automakers and parts companies achieve win-win cooperation, it makes much sense to study the current model of correlation between automakers and parts manufacturers in the world's major automobile countries, and further identify the characteristics, shortcomings and referencing-worth advantages of different "assemblycomponents" relationship models at home and abroad.

\section{Literature Review}

At present, many studies have explored the relationship between vehicle manufacturers and component suppliers (referred to as "assembly-components" relationship) in the Automobile industry. In addition to the coordination of all links in the industry chain, the long-term development of the automobile industry chain is more dependent on consistently attracting foreign auto companies with local advantages and realizing their localization [1]. As of now, it is generally believed that the most common internal risks faced by the automobile industry chain are delayed delivery by related companies and parts supply issues [2], while its most common external risks include force majeure, government regulation changes, linkage mode, supply disruption and resource constraints etc. [3]

After understanding the risks face by the autom ${ }^{1}$ obile industry chain, we can take a look at the development of "assembly-components" relationship as a vital link in the automobile industry chain. The development process of "assembly-components" relationship in the automobile industry chain can be roughly divided into three stages: The first stage happened in the global great development of automobile Industry from 1950 s to 1970 s and is represented by the vertical integration model in which vehicle manufacturers continue to extend their businesses to component manufacturers in order to reduce $\mathrm{R} \& \mathrm{D}$ costs, and component manufacturers also begin to actively rely on vehicle manufacturers in order to obtain stable orders, resulting in both sides gaining higher profit margins [4]; the second stage is represented by the traditional market competition model in which vehicle manufacturers try every means to transfer market pressure to component manufacturers, including forcing further price cuts by selecting two or three suppliers to supply the same component, and this method may eventually lead to varying degrees of supply problems[5]; the third stage is represented by the cooperation-based subcontracting model in which vehicle manufacturers and parts manufacturers are more independent from each other due to higher degree of specialization and division of labor. In the third mode, vehicle manufacturers are focused on the development of assembly technology, as well as the

\footnotetext{
*Corresponding author: 19120568@bjtu.edu.cn
} 
development and production of complete vehicles/powertrains, leaving certain other production and R\&D tasks to be completed by the parts manufacturers, who can then become highly specialized in the production of certain components and start mass production of such components to meet the needs of vehicle manufacturers around the world [6].

By reviewing the development history of the "assembly-components" relationship in the automobile industry chain, in previous studies different scholars have defined the characteristics of the relationship model of automobile industry chain participants in each region in different ways. The relationship model widely applied in Europe is parallel supply, which is based on market competition and has no clear interrelationship constraints [7], but there are also some all-round Europe-based automakers that have a high degree of vertical integration and mainly use self-made parts[8]; in the United States, the current relationship between automobile manufacturers and parts manufacturers is similar to a strategic alliance, in which first-tier supporting manufacturers provide OEMs with integrated system services and assume some administrative functions[9]; in Japan, the automobile manufacturing industry shows a pyramid-like organization structure, in which automakers, as the leader, attract small and medium-sized parts manufacturers to join the industry, and maintain cooperative relations through unique management methods which also helps form a two-way monopoly [10].

Starting from the potential risks faced by the Automobile industry chain itself, this paper summarizes the "assembly-components" relationship that plays a key role in the industry chain. With reference to the development trend of this type of relationship and the previous literature's analysis of different regional relationship models, this paper tries to interpret the "assembly-components" models in various regions from a new perspective.

\section{Pattern Analysis}

\subsection{Ford in the United States - blood ties}

Ford is a world-famous automobile brand. Ford Motor Company is one of the largest automobile manufacturers in the world, ranking 45th in the 2018 World Top 500 Brand List. Visteon is a world-renowned auto parts integration supplier, designing and developing auto parts products such as dashboards, display screens and cockpit electronic products for major auto manufacturers around the world. Visteon broke away from Ford Motor Company [11] in 2001 and became an independent listed company. Previously, the partnership between Ford and Visteon was established within the framework of vertical integration. Parts manufacturers rely more on OEM manufacturers, and the development of parts factories is restricted by OEM manufacturers. Low efficiency has led Ford to separate its Visteon company, and the two companies gradually developed into contract companies with "blood ties".
Features: 1) Similar corporate culture and development strategy. As parts enterprises are separated from the OEM enterprises, they share certain similarities in corporate culture, overall development, and corporate vision; 2) High matching degree. The OEM enterprise and the parts enterprise share "blood ties" and have high matching degree of cooperation. Thus, OEM enterprises prefer parts enterprises that share "blood ties" with them.

Strengths: 1) Stable supply relationship. OEM enterprises and parts enterprises that have high matching degree are of stable supply relationship, and share the same destiny. 2) Same strategic choices. After the separation of OEM enterprises and parts enterprises, they gradually establish a sound symbiotic relationship [12], and form a brand-new strategic partnership to jointly solve problems of price, quality and product innovation; 3 ) OEM factories feature less investment, and short product research and development cycle. OEM manufacturers can divest huge assets used for production, reduce investment in research and development and equipment, enable module suppliers to assume more responsibilities in technology and capital investment, share risks and speed up the research and development cycle [13].

Weaknesses: 1) Poor innovation of parts. Too stable a cooperative relationship may lead to poor innovation of new products, high dependence on OEM manufacturers, and easy occurrence of product updates; 2) Low profits of spare parts manufacturers. OEM manufacturers generally choose one or two parts factories. In this regard, parts manufacturers can only increase investment and inventory, which further results in higher costs and lower profits.

\subsection{Volkswagen of Germany - contractual relationship}

Volkswagen Group in Germany, the largest automobile manufacturer in Europe, is a multinational automobile group with production bases in many countries, ranking first among the top ten automobile companies in the world. Bosch is one of the German industrial enterprises, with auto component and industrial products as its core products. Bosch is well-known for its innovative and cutting-edge products and systematic solutions. Volkswagen and Bosch established their automobile industrial chain on the basis of market-oriented transaction and cooperation, with their association featuring the two-way and multi-round market-oriented transaction [14].

Features: 1) Competition among parts enterprises. Due to the similarity of production and technology, component manufacturers, as the same kind of symbiotic units, crowd and compete with each other in the market fiercely [14]; 2) Market transaction relationship. The OEM enterprises and parts enterprises established the automobile industrial chain under the background of mutual cooperation and market-oriented transaction between enterprises, with their association featuring the two-way market-oriented transaction.

Strengths: 1) Stable cooperative relationship. The OEM enterprises and parts enterprises established their long-term relationship with contracts and agreements, 
which make their cooperation stable and mature; 2) Sufficient innovation ability. The market competition system can ensure the free competition among the parts enterprises, further promoting their continuous technology improvement, thus promoting the development of the auto industry.

Weaknesses: 1) Loose connections. There is no indepth alliance between the OEM enterprises and parts enterprises. In case of any market crisis, enterprises may take measures to protect themselves under external pressure, which may hurt their former partners; 2) The profit of parts enterprise is low. OEM enterprises often make the final decision on the selection of components through bidding. In order to win the bid, parts enterprises offer a low price, which may affect their profits; 3) The fierce competition among parts enterprises. There is no long-term and stable supply between the OEM enterprises and parts enterprises, which may lead to few technical exchanges and mutual blockade among parts enterprises.

\subsection{Toyota of Japan - intersect holding}

Toyota Motor Corporation is the first automobile manufacturer with an annual output of more than 10 million sets. It is one of the top ten automobile companies in the world, and now ranks first in terms of sales volume in the world. Founded in 1949, Denso Group is the world's top supplier of auto system component, second only to Bosch Group of Germany in the component market. In the early days of its establishment, Denso grew up under the protection of Toyota, so the two companies share similar culture and gradually formed a stable supply mode in the system with property rights as their link.

Features: 1) Relationship of assets. Vertically, the OEM enterprise controls shares of the parts enterprise by tier down, while they hold shares of each other. The OEM enterprise control the small and medium-sized parts enterprises in the second and third tier through the firsttier subcontractors, forming a stable supply chain vertically; 2) Management mode. Both the OEM enterprise and the parts enterprise carry out Japanese management modes such as "Total Quality Management", "lean production" and "Kanban management"; 3) Transmission. Denso Group, as an independent entity under the framework of Toyota, usually does not need to respond to the environment directly. Changes in market conditions, competition, related industries and macro policies are more likely to affect Toyota's business decision-making, and then transmit to Denso for adjustment through mutual connection.

Strengths: 1) Strong control. OEM enterprises have strong control over parts enterprises, usually initiating technical exchange; 2) Strong collaboration and service between the OEM enterprise and the parts enterprise. The OEM enterprise will make investment in the parts enterprise. Accordingly, the parts enterprise is willing to invest a lot of manpower and resources in the early research and development to fully support the new car research and development project of the OEM enterprise; 3) Stress on personnel communication. Attention is paid to the cooperation between the technicians of the OEM enterprise and the parts enterprise, which can reduce the $\mathrm{R} \& \mathrm{D}$ cost and cycle time.

Weaknesses: 1) More likely chain reaction. These suppliers are difficult to get the business from other OEM enterprises. In case of any difficulties of the OEM enterprise, the parts enterprises will also fall into business crisis; 2) Motivation for innovation depends on the OEM enterprises. Too stable ordering between the OEM enterprise and the parts enterprise may lead to the low motivation for innovation, as well as the price problem caused by the non-transparency of the procurement process.

\subsection{Geely of China-zero competition to select the fittest}

Geely Auto Group is a leading automobile manufacturer in China, aiming to become the most competitive and respected Chinese automobile brand. In 2014, Geely launched the "Brand Simplification" strategy to complete the sales of DSI, Hunan Jisheng and Shandong Transmission successively. In the selection of auto component, Geely Auto and some core parts enterprises form a complementary and cooperative community of interests through equity participation, shareholding, share exchange and other forms. Through the global component procurement strategy, Geely Auto allows appropriate competition among its related parts enterprises and select the most suitable suppliers from global component suppliers.

Features: 1) Abundant sources. The function of the OEM enterprise is to achieve the best synergy between different sectors, and select the most suitable global parts enterprises to supply different modules after competition; 2) Large scale procurement. Geely improves efficiency and economies of scale through centralized procurement of certain parts from the company's suppliers.

Strengths: 1) Strong ability to deal with emergencies. In the face of global emergencies, the OEM enterprise can switch to manufacturers supplying similar products, granting it with strong ability to respond to safety incidents; 2) Clear definition of roles and responsibilities. The OEM enterprise has a clarified definition of internal roles and responsibilities, which can achieve the best synergy between different sectors, increase its flexibility of operation and prevent repeated investment, which helps reduce the burden of assets; 3) Small R \& D investment. Few requirements for component planning allow the OEM enterprise to reduce production and R \& D costs, shorten product development cycle, improve quality and maximize economic benefits.

Weaknesses: 1) Strong uncontrollability. Due to the economic trade friction between countries, some countries may order local parts enterprises to stop supplying for the OEM enterprise. Without core component technologies, the OEM enterprise may fall into trouble, having weak influence on the parts enterprises; 2) High costs. The OEM enterprise selects the most suitable supplier from the global supply chain. Different location of different component manufacturers will increase the logistics cost of component transportation, eventually increasing the 
price of products; 3) Low matching degree. The OEM enterprise generally choose the most suitable existing component. If customized component are required for assembly, there will be "no matching component" and a lot of manpower and resources will be required for a new
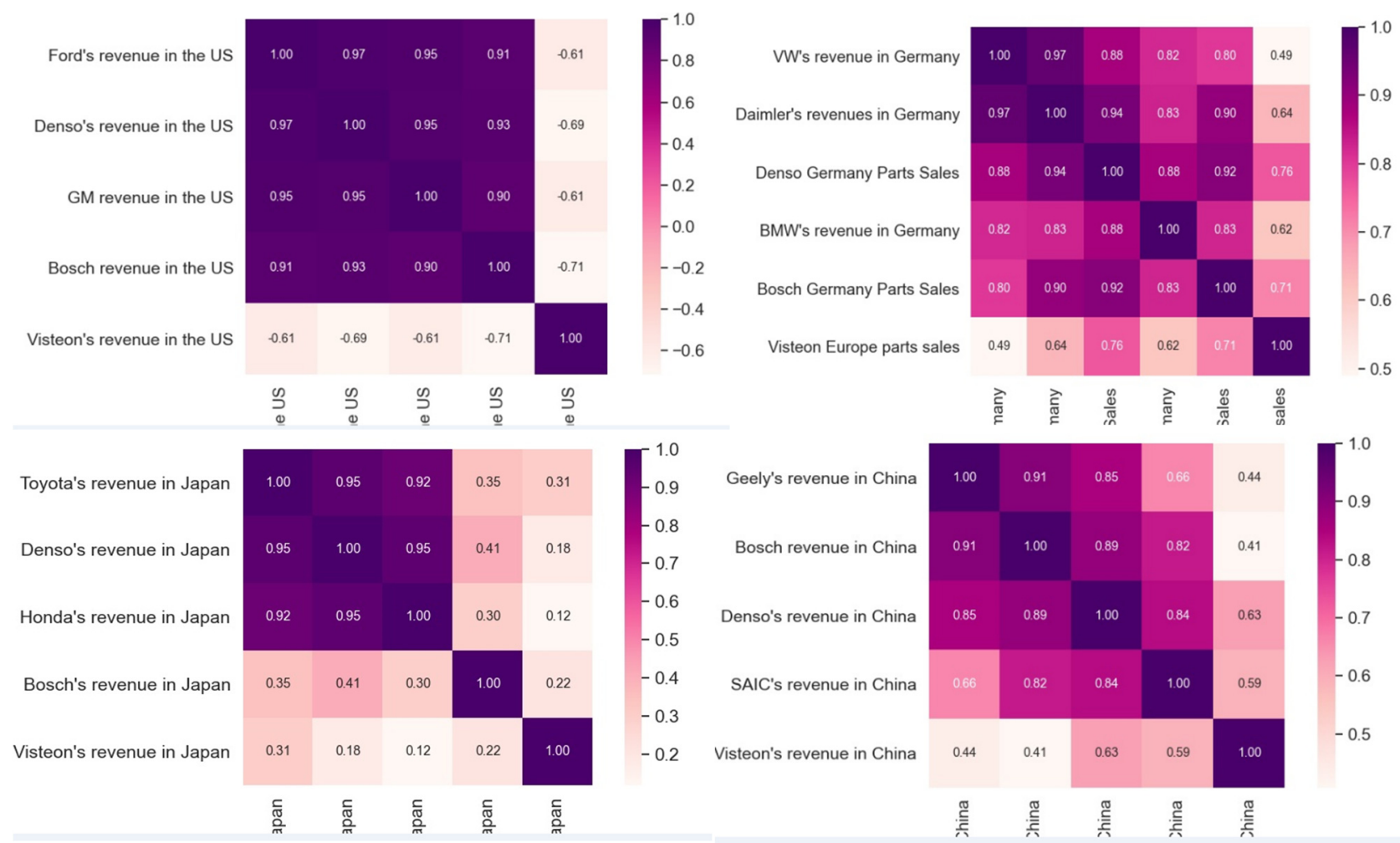

Figure 1 Correlation between automakers and parts suppliers

Correlation coefficient is a statistical indicator that measures the degree of correlation between variables and it is useful in intuitively reflecting the strategic shifts of large automakers and auto parts manufacturers in the world.

Through the correlation between Ford and various component companies (including the negative correlation between Ford and Visteon), as well as Visteon's sluggish performance in the North American market and its triumphant advancement in the Chinese mainland market in recent years, it can be seen that in the past several years Visteon has begun to shift the focus of its strategic development to the Chinese mainland market, and is trying to ease the pressure brought by high operating costs in the US market by shifting the main suppliers for orders.

Looking at the correlation coefficient between Toyota and parts manufacturers in the figure, we can see that Toyota's Japanese local business generally uses parts and components produced by Denso, and the correlation between the two is the highest. This also reflects the characteristics of the Japanese model, that is, automobile manufacturers and parts manufacturers have intertwined equity structure and they are each other's customers. In this mode, a foreign parts manufacturer can't increase its market share in Japan unless it continuously increases its investment in this market. At the same time, with Denso's heavy investment in Germany, the degree of cooperation between Denso and Volkswagen has also continued to increase. It shows that parts manufacturers from Japan round of product development.

\subsection{Correlation analysis}

have also begun to try their best to win orders in other countries and regions, in an effort to improve their innovation capabilities and profit margins.

In the Chinese market, Bosch and Geely have the highest correlation. The reason is that currently Bosch is far ahead of other parts manufacturers in terms of localization in China and its sales growth in the AsiaPacific region mainly comes from the Chinese market. In the past 20 years, despite the ups and downs in profit performance, Bosch's business in China has been profitable in general and it has been developing its business with the growth of the Chinese economy. At present, the company is still making efforts to further extending its influence in the Chinese automobile industry.

\section{Conclusion and Forecast}

Through a summary analysis of the current typical "assembly-components" relationship models, it can be seen that, the American (Ford) model has advantages including high degree of "assembly-components" pairing and stable supply relationship, and faces the possible risk of poor innovation by parts manufacturers; The German (Volkswagen) model shows a stable "assemblycomponents" partnership that is rooted in market transactions, and faces the possible risk of fierce internal competition affecting the profit margins of parts manufacturers; The Japanese model (Toyota) is 
characterized by intertwined equity structure and strong synergy of "assembly-components" companies, but this mode may lead to a situation where the two companies always "prosper and fall together"; The Chinese (Geely) model is characterized by clear division of labor between "assembly-components" companies, cost reductions brought by large-scale procurement and strong anti-risk capabilities, but low degree of "assembly-components" matching in highly customized assembly process could be a problem for those in the Chinese auto industry.

The main innovation of this research lies in interpreting from a new perspective the characteristics, advantages and disadvantages of the current "assemblycomponents" relationship models in major automobile countries. However, the correlation analysis data used in this study is limited to company sales figures in the typical "assembly-components" relationship models, and we only managed to collect some easy to quantify public data. If we could add more parameters (data dimensions) to the study, it should've made the prediction results more credible. Future research should consider using more descriptive statistical methods and other techniques to further explore the essential differences between different "assembly-components" relationship models.

\section{Acknowledgments}

Thanks to the Major Project of the Chinese Academy of Engineering (2020-HYZD-8-11) for funding this paper.

\section{References}

1. Hahn T, Auktor G V. The effectiveness of Morocco's industrial policy in promoting a national automotive industry [M]. Discussion Paper, 2017.

2. Hudin N S, Hamid A B A, Chin T A, et al. Exploring Supply Chain Risks among Malaysian Automotive SMEs [J]. International E-Journal of Advances in Social Sciences, 2017, 3(8): 666-674.

3. Kaviani M A, Tavana M, Kowsari F, et al. Supply chain resilience: a benchmarking model for vulnerability and capability assessment in the automotive industry $[\mathrm{J}]$. Benchmarking: An International Journal, 2020.

4. Li Surong. Discussion on Organization Structure Mode of Automotive Complete Vehicle Industry and Component Parts Industry [J]. Auto Industry Research, 2001(11):23-27.

5. Chen Ying, Wang Cunyu, Dong Han, Cao Wenquan. Research on Customized Steel and Parts for Automotive Body-in-White [J]. Journal of Automotive Engineering, 2016,6(04):302-305.

6. Cai Changshu. Supply Chain-based Research on Purchasing Management of Auto Parts [D]. Wuhan University of Technology, 2005.

7. Zhang Zhengzhi. Analysis on the Development of Supply Chain Relationship In China's Auto Industry [J]. Automotive Research and Development, 2005(10):2-8.

8. Wang Kun. Industrial Chain-based Research on the
Interactive Development of "Assembly-Components" Relationship in the Auto Industry [D]. Hefei University of Technology, 2010.

9. Liu Zhiying, Wang Kun. The "Complete Vehicle and Parts" Cooperation Pattern of US Auto Industry and Its Implications [J]. Auto Industry Research, 2009(07):45-48.

10. Zhang Qian, Dai Long. The "Complete Vehicle and Parts" Cooperation Pattern of Japanese Auto Industry and Its Implications [J]. Global Science,Technology and Economy Outlook, 2014, 29(08): 72-76.

11. Wu Qitang. A Section of Arrowroot and the Fibres-What is the Truth behind the Separation of Visteon and Ford? [J]. Automobile\&Parts, 2005(24):39-41.

12. Liu Zhiying, Wang Kun. The "Complete Vehicle and Parts" Cooperation Pattern of US Auto Industry and Its Implications [J]. Auto Industry Research, 2009(07):45-48.

13. Lin Jihong. Modular Production and the Evolution of the "Assembly-Components" Relationship in the Global Automotive Industry [J]. World Economic Research, 2013(07):3-7+87.

14. Sun Xiaohua, Qin Chuan. Governance Model for Longitudinal Relationship of Industrial Chain Based on the Symbiosis Theory: Comparison and Enlightment of U.S., European and Japanese Automotive Industries [J]. Economist, 2012, 3(3): 95102. 